\title{
Central Asia on the Cloud: Prototyping an Open Regional Atlas
}

\author{
Ainura Nazarkulova and Josef Strobl \\ Interfaculty Department of Geoinformatics - Z_GIS, University of Salzburg, Austria · \\ ainura.nazarkulova@sbg.ac.at
}

Short paper

\begin{abstract}
The first generation of digital, and subsequently online, regional atlases did only little to reconceptualize the idea behind this established cartographic category. Using the case study of a regional atlas for Central Asia, the authors propose a fundamental re-thinking as a usercentric and target-group-specific front end for emerging Spatial Data Infrastructures. 'Next generation' regional atlases are anticipated as open frameworks based upon distributed online services; extensible, mashable, and modifiable, flexibly adjusting to situated devices.
\end{abstract}

\section{Introduction}

Regional atlases (see ICA Commission on Atlases - http://icaci.org/commissions) follow a long tradition and are considered an established category of cartographic publishing. They typically serve the dual purposes of (i) general education, and (ii) spatially-oriented repositories of regionalized information, although often primarily considered scientific publications (e.g. MOSER 2006). Like all maps in the pre-digital area, atlas cartography had to strike a compromise between serving as storage as well as presentation media for spatial information. Frequently, regional atlases were developed due to underlying intents to establish, strengthen and popularize regional identities. In Central Asia, this is well illustrated by the fact that the impressive tome 'Atlas of the Kirghiz Soviet Socialistic Republic (Kirghiz SSR)', published during the Soviet era in 1987, was re-labeled with front cover stickers as the 'National Atlas of the Kyrgyz Republic' after independence, now to communicate the identity as a nation.

Compiling, designing and publishing a regional atlas traditionally amounted to a major effort, spanning years if not decades. Cartographic experts working with teams of domain specialists were frequently organized within major projects, which even led to the establishment of permanent departments (e.g. Tirol Atlas). Regional atlases created through once-off efforts ('projects') tend to suffer from the lack of continuity, updates, and maintenance of all time-dependent or newly acquired information.

Regional or national atlases rarely followed established principles or modelled after some blueprint, but were often tied to research initiatives resulting in unique and even outstanding products. Examples are the Salzburg-Atlas (LENDL et al. 1955), pioneering the use 
of iconic symbols in statistical maps, or the Tirol Atlas (FÖRSTER 2003), demonstrating the cartographic potential of SVG for online maps.

Due to this leading-edge tradition in cartography, regional atlases - in particular in Central Europe - turned into early experimenters and adopters of digital and online technologies. As with other 'digital cartography' efforts, these were mainly driven by available visualisation techniques on digital and online platforms, and tended to be measured against the graphic quality of print cartography. The more fundamental step of aligning the atlas concept with current distributed computing environments yet needs to be taken, though - even if it might mean that 'regional atlas' switches from a product to a metaphor for a web portal.

\section{Target Audience}

The ways of using atlases and maps are changing along with changing media and enabling technologies, ranging from the Internet as such, to embedded code facilitating individuals' exploration of information about regionalized topics. Due to the much more flexible and interactive character of interfaces, more and different users beyond the traditional groups of pupils, students, and an interested, educated general audience are anticipated.

Any communication product needs to be designed starting from the needs and expectations of its intended users. The target audience of an online regional atlas is obviously varied, and changing and growing with technology development and more people having access to the Internet, and thus to online atlases. The target audience is likely changing too, due to a different demographic of e.g. younger people accessing the Internet and related technologies. There is no hard evidence, though, that younger and affluent people interact more with online atlases in everyday life.

Specific target groups for any particular regional atlas need to be established up front, and are typically co-determined by the origin and context of any such project. A general, broad audience will be addressed when political identity is emphasized, or formal learners in schools and universities when an atlas is considered primarily an educational tool. (Too) frequently an atlas is not really demand driven, but based on existing supply, e.g. when serving as an outlet for a statistics agency. Whenever a particular theme and specific region are combined, target groups are typically well understood (e.g. SCHWEIKART et al. 2014).

The concept of 'next generation' atlases outlined in this paper aims - inter alia - at avoiding the conflicts of interest arising from mixed target groups. Slim and lightweight atlases built upon open and distributed architectures allow for atlas interfaces individualized for specific and narrow target groups, much like targeted 'apps' instead of multi-purpose heavy-duty applications.

\section{Online Atlas - Revisited}

\subsection{First Generation Digital Atlases}

Most of the Internet content, just like initial digital content, began as digital representations of established media. Databases emerged from index cards and rolodexes, word processing 
from 'smart' typewriters, and CAD from technical drafting. Maps, and collections of these organized as atlases, also initially passed through this step of a mere 'digital conversion' without too much attention to the fundamental differences inherent to digital media.

Digital (school) atlases, sometimes referred to as 'Atlas Information Systems' (HURNI 2006), first emerged from scanning or save-as-image processes and were distributed on CDs just like national maps were converted to digital applications. Map sheets, inherent scales and fixed designs were mostly retained, while the advantages from digital conversion were evident in the search function, the possibility of annotation, and easier access. In some cases, perspective viewing ('3D'), or even virtual globe capabilities (STROBL 2006), were included to enhance attractivity. To retain the advantages of both print and digital or even online media, 'companion applications' were developed by several publishers.

Subsequently, increasingly sophisticated web technologies were brought to fruition. Moving from document centric maps in image or pdf formats (as demonstrated, e.g. in the Socio-Economic Atlas of the Lao PDR, http://www.laoatlas.net) towards more dynamic platforms, leveraging e.g. SVG, increasingly interactive atlases were published. Leading examples are the 'ÖROK-Atlas' (SEIDL \& KRIZ 2006, http://www.oerok-atlas.at), 'Tirol Atlas' (AISTLEITNER 2009, http://tirolatlas.uibk.ac.at), or 'Atlas der Schweiz' (SIEBER et al. 2011, http://www.atlasderschweiz.ch).

These products offered seamless access and scalability within the respective target region, while replacing graphics with geo-graphics. Flexibility regarding design and visualization, extensive query capabilities including individualized charts, contrasting (feature comparisons), and reporting are typical characteristics

Still, these highly attractive and feature-rich atlases adhere to the concept of a 'closed' product, fully controlled by authors. Reference (base) maps and thematic layers are predetermined, as is all functionality and the overall access framework. Use of atlas content in another environment, as well as the integration of additional data into the atlas, or independent presentation of atlas contents are rarely feasible.

\subsection{Spatial Data Infrastructures and Atlases}

Some authors differentiate Spatial Data Infrastructures by their regional extent (MASSER, 2005), following a global - regional - national - etc. hierarchy. This suggests a parallel with (regional) atlases. The authors consider a 'next generation' regional atlas as an enduser oriented and often domain specific interface for a (regional) SDI, just as a geoportal serves as a professional or 'geospatial expert' interface for an SDI.

This approach implies that data management and other back-end functionality like base maps, or search and discovery, are no longer a core part of an atlas, but supplied by an SDI framework. Atlas design is thus primarily focussing on providing a user experience for the intended target audiences.

Regional and other (e.g. thematic) atlases are therefore bound to be more flexible, scalable, and open - as an online resource to tie into, to work with, to analyse, re-purpose, and to integrate into other products, instead of existing as an encapsulated entity as suggested by traditional atlas concepts. By leveraging the power of SDIs, atlas developers can more fully 
concentrate on their communication tasks, coupling the presentation interface to the SDI backend through open service specifications (BUEHLER \& MCKEE, 1996).

\subsection{Next Generation Regional Atlas}

Following the approach outlined above, atlases can be designed as open platforms. While the 'open' concept might be applicable here in the sense of open access and licensing (ENGELFRIET 2010), an 'open atlas' can be characterised with criteria like:

- All content is documented to be accessible via web services following open standards specifications.

- The atlas extent (region of interest) is not strictly enforced; global reference maps provide the backdrop for flexible handling of cross-boundary themes.

- The thematic scope is extensible, by accessing other web services and fusing these into the atlas interface.

- Features and themes are fully accessible from a linked open data perspective (Tim Berners-Lee - http://5stardata.info). Linking options of course include non-spatial data, e.g. dictionary entries or ontological frameworks.

- Symbolization can be freely adjusted by users, starting from sensible defaults adhering to established cartographic principles.

- Overall, atlas contents are 'highly mashable', tearing down the boundaries between any specific atlas and other potentially related information.

Any atlas concept adhering to this architectural framework will perhaps use a name and interface building upon end users' traditional understanding of an 'atlas', but ultimately offers a much more powerful and open approach. A regional atlas defined as a regional and possibly thematic interface is primarily an access node for a defined community (target audience), avoiding any sharp delineation in

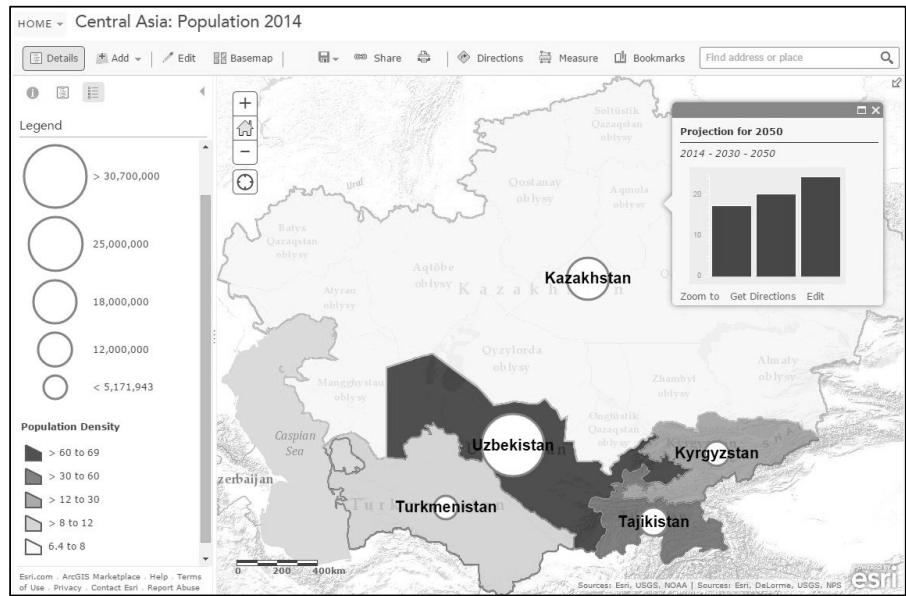

Fig. 1: Regional overview sample map geographic, thematic, or temporal terms.

'Atlases' of this kind can easily overlap, evolve, branch out and invite versioning. This of course brings up issues of curation, authority, quality, and validity. Following the proposed focus on interfacing, these questions are relegated to the underlying SDI, though. 


\section{Cloud and Services as the Platform}

According to the concept outlined above, an atlas is considered a user- and demandoriented, regionally and/or topically defined access portal to web services, provided by numerous different institutions. One example of the power of services-based architecture is the increasing inclusion of satellite or aerial imagery, popular as reference maps for presenting information in online atlases.

Following the principle of decentralized hosting of services at their respective 'home' institutions, the current trend towards cloud computing provides a flexible and powerful infrastructure for SDI, as well as its interfaces - 'atlases' for regional or thematic domains and 'geoportals' for geospatial (infrastructure) professionals. Essentially, only the emergence of cloud computing turned the

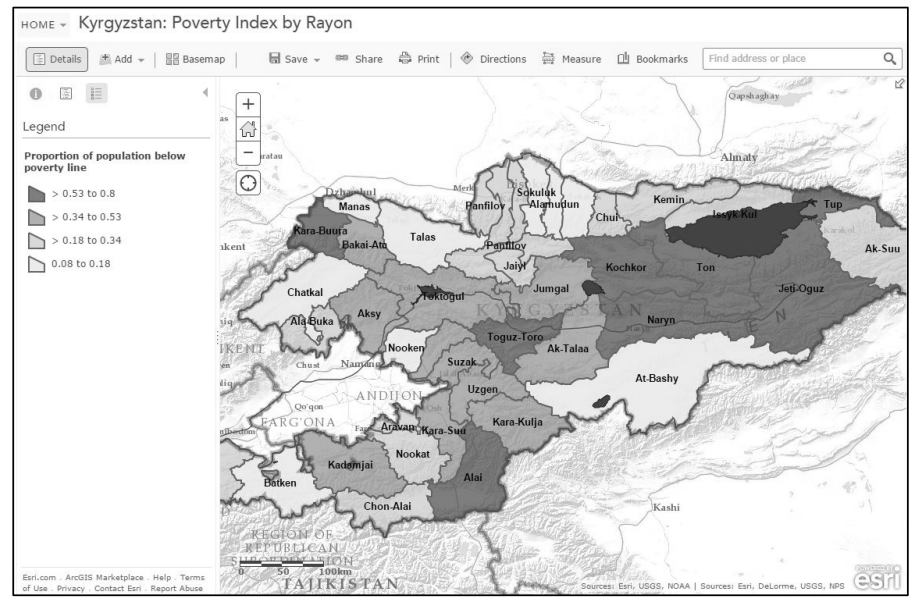

Fig. 2: Country level statistics sample map Internet with all its distributed components into a true infrastructure, by decoupling the location, operators, and dimensions of services provisioning from services use.

All this only becomes feasible through open standards, predominantly developed within the Open Geospatial Consortium, playing a decisive role in distributed online atlas architectures. These facilitate the move from 'heavy' centralized atlas platforms towards flexible, even ad-hoc provision of atlas-like services featuring live maps e.g. through storymapping (WRIGHT 2014).

\section{$5 \quad$ Insights and Perspectives}

Traditional atlases were collections of static maps or maps with static information. With digital online atlases, users not only access static information created or curated by authors, but explore changes and dynamics of any phenomena of even very narrow interest, for example providing the backdrop and contextualisation of specific mobility patterns - as demonstrated in http://taxi.imagework.com. Crowdsourcing, prosumerism, participation, and social media link into this revised atlas concept.

Online atlases, when transitioning above and beyond merely 'digitizing' traditional formats and fully leveraging interaction, multiple media, and orchestrated services, are to be considered a significant paradigm shift, with the traditional term of 'atlas' being used more 
metaphorically than literally on yet another level. The re-thinking and re-engineering of approaches to regional atlases has only begun.

\section{References}

AisTleitNeR; J. (2009), Der Tirol Atlas - Stand und Weiterentwicklung eines modernen, länderübergreifenden Regionalatlas. Kartographische Nachrichten -Fachzeitschrift für Geoinformation und Visualisierung, 1/2009, 18-25.

BueHler, K. \& MCKeE, L. (Eds.) (1996), The OpenGIS Guide: Introduction to Interoperable Geoprocessing: Part I of the Open Geodata Interoperability Specification (OGIS). Open GIS Consortium, Inc.

Engelfriet, A. (2010), Choosing an Open Source License. Software, IEEE, 27 (1), 48-49.

FÖRSTER, K. (2003), Tirol Atlas: An SVG based online Atlas Information System. In: Proceedings SVG Open 2003 (Web), Vancouver 2003.

HURNI L. (2006), Interaktive Atlasinformationssysteme - quo vaditis? Kartographische Nachrichten, 3/2006, 136-142.

Lendl, E., Pfitzner, W. \& Willvonseder, K. (Eds.) (1955), Salzburg-Atlas. Bundesland Salzburg in 66 Kartenblättern. Otto Müller, Salzburg, 2 Bände.

MASSER, I. (2005), GIS worlds: creating spatial data infrastructures. Esri Press, Redlands.

Moser, J. (2006), Der „Atlas zur Geschichte und Landeskunde von Sachsen“: Ein thematischer Regionalatlas. In: Kartographie als Kommunikationsmedium. Wiener Schriften zur Geographie und Kartographie, 17. Wien.

Schweikart, J., Franke, C. \& Henke, S. (2014), Atlas of health infrastructure for the Mbeya Region in Tanzania - Regional atlases as information source using geoinformation systems. Journal of Maps, 10 (4), 620-629. Taylor \& Francis.

SEIDL, M. \& KRIZ, K. (2006), ÖROK-Atlas online - Atlas Informationssystem Austria (AIS) - Ein interaktives multimediales Informations- und Analyseinstrument. In: 11. Int. Konf. zur Stadtplanung u. Reg. Entwicklung, ed. by. M. Schrenk, Verein CORP, Wien.

Sieber, R., Hollenstein, L. \& Hurni, L. (2011), Der neue Atlas der Schweiz 3 auf DVD. Kartographische Nachrichten, 61 (2), 86-89.

Strobl, J. (2006), Der Globus ist des Atlas Tod. In: Jekel, T., Koller, A. \& Strobl, J. (2006), Lernen mit Geoinformation. Wichmann, Heidelberg, 2-10.

Wright, D. (2014), Speaking the "Language" of Spatial Analysis via Story Maps. Blog entry (Sept. 14, 2014). http://blogs.esri.com/esri/esri-insider/2014/09/15/speaking-thelanguage-of-spatial-analysis-via-story-maps/. 